\title{
EXACERBACIÓN APICAL POSTERIOR A TRATAMIENTO ENDODÓNTICO
}

\author{
${ }^{1}$ Claudia Liliana Martínez Vera, ${ }^{2}$ Liliana Rodríguez Ballesteros, ${ }^{3}$ Antonio Díaz Caballero \\ ${ }^{1}$ Odontóloga Fundación Universitaria San Martín, Estudiante Especialización en Endodoncia U. de Cartagena, Colombia. ${ }^{2}$ Odontóloga U. de Cartagena, \\ Estudiante Especialización en Endodoncia U. de Cartagena, Colombia. ${ }^{3}$ Odontólogo U. de Cartagena, Especialista en Periodoncia U. Javeriana, Magíster en Educación U. del Norte, \\ Candidato a Doctor en Ciencias Biomédicas U. de Cartagena, Profesor titular U. de Cartagena,Colombia.
}

Autor responsable de correspondencia: Claudia Liliana Martínez Vera

Correo electrónico: odontomartinezvera@yahoo.es

\section{RESUMEN}

Un tratamiento endodóntico genera muchas expectativas en el paciente debido a la creencia popular de ser un tratamiento incómodo, doloroso y tensionante que demanda varias sesiones para su realización. Cuando el diagnóstico pulpar es sintomático, estas expectativas aumentan y lo más deseable para el profesional es que el tratamiento sea superado, es decir, eliminar toda la sintomatología para garantizar el éxito de la terapia. Las agudizaciones endodónticas son complicaciones que se presentan durante la realización un tratamiento endodóntico al formar procesos inflamatorios periapicales que se presentan al finalizar el tratamiento. Algunos factores influyen significativamente en el desarrollo de la exacerbación de una endodoncia como el diagnóstico pulpar, la presencia de tractos fistulosos, instrumentación extensiva, única cita y falta de instrumentación y de irrigación, entre otros. Este artículo describe el caso clínico de un premolar superior con un diagnóstico inicial de periodontitis apical crónica no supurativa, sometido a un retratamiento endodóntico, el cual presentó un error en la preparación por subinstrumentación al determinar una longitud de trabajo insuficiente que ocasionó una limpieza deficiente y una lesión apical. [Martínez CL, Rodríguez L, Díaz AJ. Exacerbación apical posterior a tratamiento endodóntico. Ustasalud 2012; 11: 130 - 134]

Palabras clave: Irrigación terapéutica, Instrumentación, Periodontitis periapical

\section{APICAL EXACERBATION AFTER ROOT CANAL TREATMENT}

\section{ABSTRACT}

An endodontic treatment generates high expectations for the patient due to the popular belief treatment be uncomfortable, painful, stressful it takes several sessions to complete it. When the pulp is symptomatic, these expectations rise and the professional expects to finish and to remove all the symptoms to ensure the success of therapy. Endodontic exacerbations are complications than can be presented during endodontic treatment to form periapical inflammatory processes that occur at the end of treatment. Some factors influence the development of these complications such as pulp diagnosis, the presence of sinus tracts, extensive instrumentation, lack of instrumentation and irrigation, among others. This article describes the case of a premolar with an initial diagnosis of chronic suppurative apical periodontitis, endodontic retreatment under which introduced an error in the preparation by subinstrumentación to determine a working length that caused insufficient and inadequate cleaning apical injury.

Key words: Recurrence, Therapeutic irrigation, Instrumentation, Periapical periodontitis

Recibido para publicación: diciembre 16 de 2011. Aceptado para publicación: junio 30 de 2012

\section{INTRODUCCIÓN}

La agudización endodóntica se define como la complicación de dolor y/o inflamación que hace que un paciente consulte de manera imprevista y que sorprende tanto al profesional como al paciente, también llamada exacerbación aguda. ${ }^{1-3}$ Desde 1960, diversos autores definen agudización según el origen y la sintomatología que refiera el paciente. ${ }^{4}$ Una exacerbación se refiere al dolor postoperatorio y/o inflamación resultante de la irritación bacteriana, mecánica o química. El diagnóstico y tratamiento oportuno son esen- ciales para reducir el dolor y el malestar del paciente. $^{5}$

Los factores clínicos se clasifican en tres grupos: ${ }^{2,3,6,7}$

- Irritantes: se encuentran dentro del conducto radicular como el tejido pulpar necrótico, las bacterias, los subproductos bacterianos, sustancias nocivas y antígenas.

- Factores terapéuticos iatrogénicos durante la terapia endodóntica: se señalan las soluciones irrigadoras, la sobre y subinstrumentación, hiperoclusión, debridación incompleta y medicamentos colocados dentro del conducto. 
- Huésped: se encuentra la edad, sexo, presencia de dolor preoperatorio, presencia de fístula y tamaño de la lesión apical.

Prevenir el paso de estos factores resultará la mejor medida para evitarlos, con técnicas de limpieza y conformación que ayuden a extruir menos restos al periápice.

La incidencia de las agudizaciones es variable. ${ }^{8} \mathrm{~A}$ partir de 1987, la incidencia de agudizaciones alcanza un $20 \%$ al tener en cuenta la inflamación como base, después de tratar pulpas necróticas y presentándose el paciente antes del tratamiento asintomático con una imagen radiolúcida al final., ${ }^{3,9-11}$

No existen diferencias en términos de éxito radiológico entre tratamientos de conductos radiculares de visita única y de visitas múltiples. Gran parte de las complicaciones de corto y largo plazo son similares en términos de frecuencia aunque los pacientes sometidos a una sola visita pueden experimentar una mayor inflamación y son más propensos a utilizar analgésicos. ${ }^{12}$

Los factores relacionados con la incidencia de agudizaciones son edad y sexo del paciente, diente involucrado, historia de dolor preoperatorio, presencia y tamaño de la lesión periapical, inflamación preoperatoria, repetición del tratamiento endodóntico, número de citas, sobreinstrumentación, historia de alergia sistémica, enfermedades sistémicas y técnica de instrumentación, entre otros. Para cada uno de estos factores existe una opinión diferente. A continuación se revisan algunos de ellos. ${ }^{2,6,13-15}$

Inflamación preoperatoria: la presencia de inflamación preoperatoria, localizada o difusa, tiene una incidencia del $15 \%$ de los casos estudiados. ${ }^{16}$

Presencia de dolor preoperatorio: los casos de pulpas no vitales con dolor preoperatorio presente al momento de la cita inicial tienen más probabilidades de presentar dolor postoperatorio que los casos de pulpas vitales, independientemente que éstas presenten o no, dolor preoperatorio. Las probabilidades disminuyen en los casos de pulpas necróticas asintomáticas, el factor más importante relacionado con la incidencia de agudizaciones ha sido la presencia de dolor preoperatorio severo, el cual ocasiona el 19\% de incidencia. ${ }^{8,13,17-19}$

Técnicas de instrumentación: ninguna técnica es efectiva en prevenir la extrusión de restos a través del foramen apical, pero sí se ha reportado que es mayor la cantidad de los mismos cuando se utiliza la técnica de paso atrás. ${ }^{14,20-22}$

Número de citas: no se encuentran diferencias en la incidencia de dolor postoperatorio existente entre los tratamientos endodónticos en varias citas y aquellos realizados en una sola cita. ${ }^{1,23-25}$

Sobreinstrumentación: ha existido una gran controversia acerca del límite apical hasta el que debe llegar la instrumentación de los conductos radiculares, cuando los dientes se encuentran asociados con lesiones periapicales. Si ocurre, es probable que sea el resultado de una instrumentación vigorosa que ha transportado microorganismos, restos dentinarios o ambos, a través del foramen apical manipulados por el ápice de un diente infectado, éstos provocan gran cantidad de daño tisular. Los tejidos periapicales traumatizados sirven como nido para el crecimiento y multiplicación de los microorganismos. ${ }^{2,16,20}$

Repetición del tratamiento endodóntico: se señala una mayor incidencia de agudizaciones en dientes que requieren la repetición del tratamiento endodóntico Debido a que al tratar de retirar la gutapercha contenida dentro del conducto, ésta tiende a extruir las bacterias y detritus hacia el periápice, donde causarán inflamación aguda y dolor severo. Al igual que el solvente utilizado puede contribuir con un incremento en la respuesta inflamatoria. ${ }^{1,2,13}$

\section{REPORTE DE CASO}

Paciente de género femenino, de 52 años, sin antecedentes médicos de importancia que consulta al odontólogo general por presentar proceso fistuloso a nivel apical del órgano dental del segundo premolar superior izquierdo (25), en mayo de 2011. El diagnóstico fue absceso apical crónico en el 25 y fue tratada con Amoxicilina de $500 \mathrm{mg}$ cada ocho horas por siete días, luego se remitió a consulta por endodoncia para realizar el tratamiento (Figura 1).

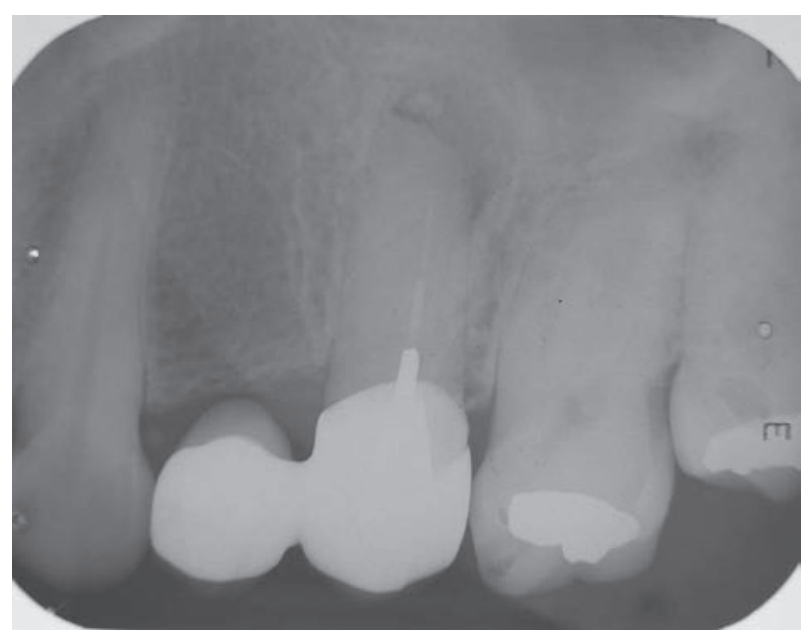

Figura 1. Radiografia preoperatoria, nótese la endodoncia deficiente con solo un conducto obturado y lesión apical. 
El 3 de junio, la paciente, consulta en la Especialización de Endodoncia de la Facultad de Odontología de la Universidad de Cartagena, refiere que hace 21 años se le realizó terapia endodóntica y rehabilitación a nivel del órgano dental del 25 y, eventualmente presentó episodios de fistulizaciones sin tratamiento. En el examen clínico no presentó sintomatología, ni proceso fistuloso a nivel apical del 25. Radiográficamente, se observó el 25 con terapia endodóntica deficiente y un solo canal tratado con lesión apical asociada. En ese momento, se diagnosticó una periodontitis apical asintomática y el tratamiento consistió en retratamiento endodóntico del 25 (Figura 2).

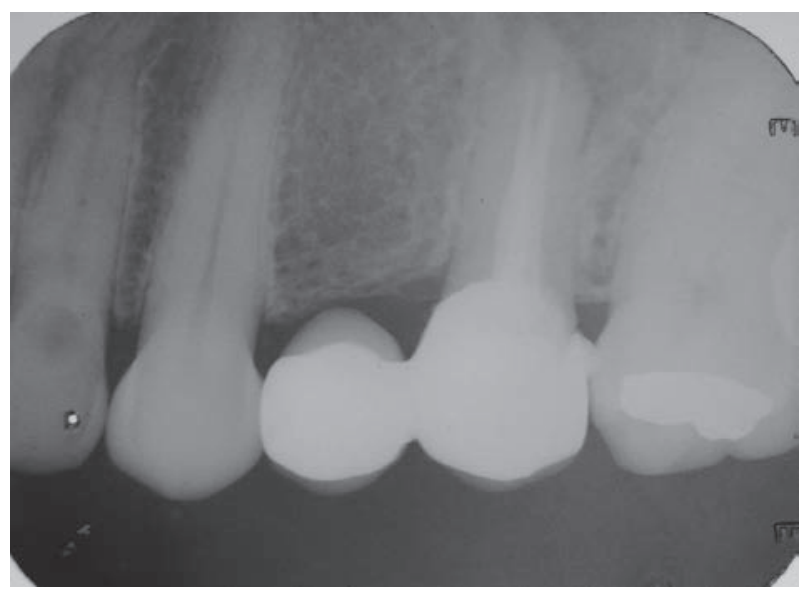

Figura 2. Radiografia postratamiento donde se observan dos conductos obturados.

Se anestesió con anestocaína al 2\% con epinefrina 1:80.000 (Rophson Therapeutics Ltda.), se retiró la prótesis fija con baja puente. Se aisló con grapa y tela de caucho, se retiró el núcleo colado con fresa redonda y troncocónica delgada. Se observó gutapercha en el canal palatino y se localizó el conducto vestibular para ser tratado. El conducto palatino se desobturó con limas tipo $\mathrm{K}$, xilol en tercio cervical y medio con abundante irrigación. La permeabilidad de los conductos no se logró en el tercio apical por lo que la longitud se estableció con el localizador de conductos Root ZX (J. Morita, Japón) en $19 \mathrm{~mm}$ para el conducto palatino y $18 \mathrm{~mm}$ para el vestibular. La conformación y limpieza de los conductos se realizó con técnica manual. Los canales se irrigaron con NaOCL al 2,5\%; posteriormente, fueron secados con puntas de papel. Se realizó la radiografía de conometría y se obturó con la técnica de condensación lateral con conos (Dentsply, Maillefer, Suiza, CH-1338 Ballaigues) y como cemento sellador se utilizó Topseal (Dentsply, Maillefer). El tratamiento posterior a la endodoncia consistió en la rehabilitación del 25 con poste adhesivo X-Post (Dentsply Maillefer, Maillefer Instruments) y corona metal porcelana.
El 12 de julio de 2011, la paciente consultó nuevamente, por presentar fistula a nivel apical del 25 (Figura 3). Se decidió en acuerdo con la paciente, realizar cirugía de apicectomía y curetaje apical, la cual, se realizó el 4 de agosto (Figura 4). Los controles se realizaron en diciembre cuando se apreció la reparación apical (Figura 5).

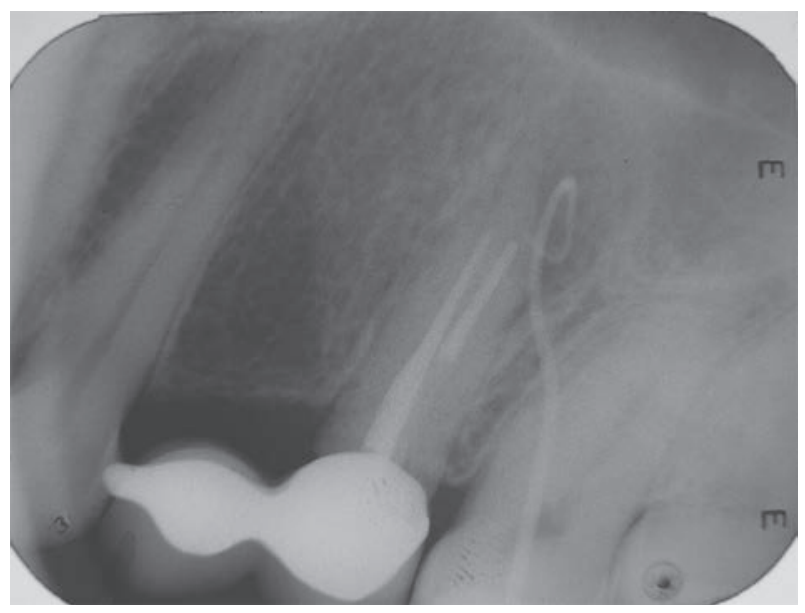

Figura 3. Radiografia de fistulografia donde se observa compromiso de la raíz palatina con lesión apical.

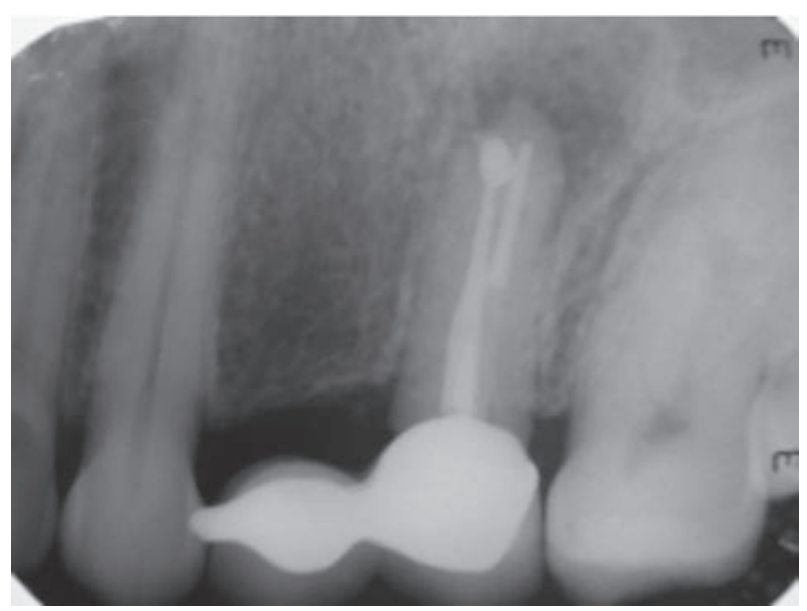

Figura 4. Radiografia posterior a cirugía de apicectomía.

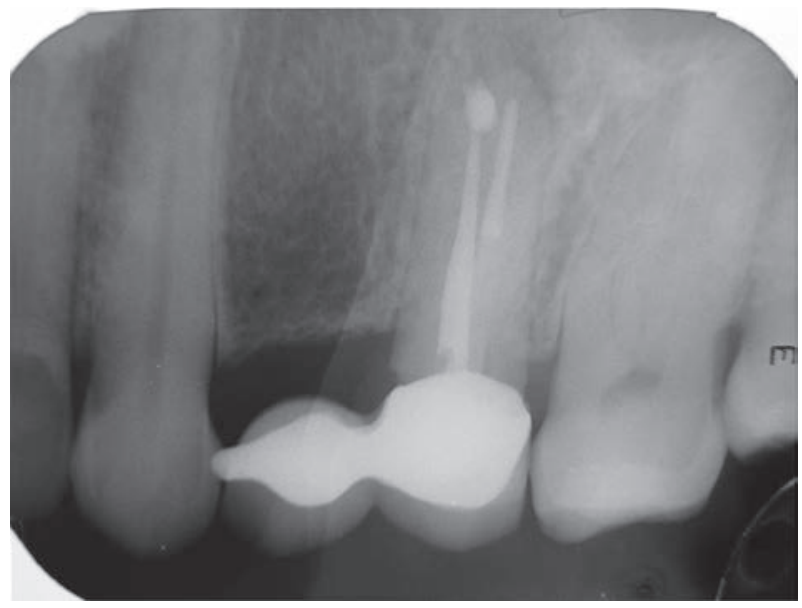

Figura 5. Radiografia de control donde se aprecia reparación de la lesión apical. 


\section{DISCUSIÓN}

En la descripción de este caso clínico se muestra un caso de agudización endodóntica posterior a un retratamiento de un diente no vital asociado con una imagen radiolúcida apical, que se encontraba asintomático y que una vez terminado el tratamiento endodóntico requirió de una nueva consulta a consecuencia de la presencia de una fístula. Hoy en día se reconoce que las razones por las cuales se producen las agudizaciones endodónticas no están claras, pero en este caso se presentaron unos factores específicos. Entre los factores que ayudaron en la agudización de este caso están la subinstrumentación apical, debido a que como lo mencionan Wu y colaboradores para los conductos infectados; la longitud de la instrumentación ideal sería estar por debajo del nivel al que las bacterias han contaminado, es decir, debajo del foramen apical, objetivo que no se superó; y, la madurez del biofilm, en este caso de veinte años lo que resulta ser directamente proporcional a su capacidad de defensa y a su resistencia a ser eliminado en dientes con necrosis de larga evolución o retratamientos. ${ }^{26}$ La relación de estos dos factores es evidente y determinante ya que la subinstrumentación o la falta de permeabilidad apical impidió que la irrigación llegara a nivel del foramen, limitó el contacto de la solución irrigadora con los microorganismos asociados a la lesión apical y produjo una debridación incompleta. Adicionalmente, la rehabilitación fue inmediata a la endodoncia. ${ }^{1}$

\section{Conclusiones}

Los diversos factores que inciden en la ocurrencia de una agudización endodóntica, llevan a sugerir las siguientes afirmaciones:

Diligenciar correctamente la historia clínica es vital para determinar si el paciente puede desarrollar una agudización endodóntica.

Luego de analizar cada una de las hipótesis planteadas por los distintos autores, es necesario reconocer que hasta el momento, la etiología de las agudizaciones endodónticas no se ha establecido por completo.

Esta situación ha hecho que se planteen diversos tratamientos para prevenir y aliviar los síntomas que se presentan durante las mismas.

Las endotoxinas bacterianas han sido consideradas como antígenos potentes capaces de producir una respuesta inflamatoria aguda. Una óptima instrumentación e irrigación permite que el conducto sea permeable para obtener una mejor eliminación de los microorganismos presentes en el ápice y así evitar que se produzca una agudización endodóntica.

\section{BIBLIOGRAFÍA}

1. Tsesis I, Faivishevsky V, Fuss Z, Zukerman O. Flare-ups after endodontic treatment: a meta-analysis of literature. J Endod. 2008; 34 (10): 1177 - 1181.

2. Iqbal M, Kurtz E, Kohli M. Incidence and factors related to flare-ups in a graduate endodontic programme. Int Endod J. 2009; 42 (2): 99 - 104.

3. Alves V de O. Endodontic flare-ups: a prospective study. Oral Surg Oral Med Oral Pathol Oral Radiol Endod. 2010;110 (5): e68 - e72.

4. Pirila V, Rantanen AV. Root canal treatment with bacitracin-neomycin as cause of flare-up of allergic eczema. Oral Surg Oral Med Oral Pathol. 1960; 13: 589 - 593.

5. Zuckerman O, Metzger Z, Sela G, Lin S. "Flare-up” during endodontic treatment--etiology and management. Refuat Hapeh Vehashinayim. 2007; 24 (2): 19 - 26, 69.

6. Sathorn C, Parashos P, Messer H. The prevalence of postoperative pain and flare-up in single- and multiple-visit endodontic treatment: a systematic review. Int Endod J. 2008; 41 (2): 91 - 99.

7. Ng YL, Mann V, Gulabivala K. A prospective study of the factors affecting outcomes of nonsurgical root canal treatment: part 1: periapical health. Int Endod J. 2011; 44 (7): 583 - 609.

8. Siqueira JF, Jr., Rocas IN, Favieri A, Machado AG, Gahyva $\mathrm{SM}$, Oliveira JC, et al. Incidence of postoperative pain after intracanal procedures based on an antimicrobial strategy. J Endod. 2002; 28 (6): 457 - 460.

9. Morse DR, Furst ML, Belott RM, Lefkowitz RD, Spritzer IB, Sideman BH. Infectious flare-ups and serious sequelae following endodontic treatment: a prospective randomized trial on efficacy of antibiotic prophylaxis in cases of asymptomatic pulpal-periapical lesions. Oral Surg Oral Med Oral Pathol. 1987; 64 (1): 96 - 109.

10. Morse DR, Furst ML, Lefkowitz RD, D’Angelo D, Esposito JV. A comparison of erythromycin and cefadroxil in the prevention of flare-ups from asymptomatic teeth with pulpal necrosis and associated periapical pathosis. Oral Surg Oral Med Oral Pathol. 1990; 69 (5): 619 - 630.

11. Matusow RJ. Endodontic cellulitis 'flare-up'. Case report. Aust Dent J. 1995; 40 (1): 36 - 38.

12. Figini L, Lodi G, Gorni F, Gagliani M. Single versus multiple visits for endodontic treatment of permanent teeth: a Cochrane systematic review. J Endod. 2008; 34 (9): 1041 - 1047.

13. Al-Negrish AR, Habahbeh R. Flare up rate related to root canal treatment of asymptomatic pulpally necrotic central incisor teeth in patients attending a military hospital. J Dent. 2006; 34 (9): 635 - 640.

14. Pi GL, Yin SH. A study of the related factors and prophylactic measures of endodontic interappointment emergencies. Hua Xi Kou Qiang Yi Xue Za Zhi. 2004; 22 (6): $471-473$.

15. Xu Q, Fan MW, Fan B, Ling JQ, Chen H, Wei X. Clinical evaluation of three nickel-titanium rotary instruments in preparation of curved root canals. Hua Xi Kou Qiang Yi Xue Za Zhi. 2005; 23 (4): 286 -288.

16. Siqueira JF, Jr. Microbial causes of endodontic flare-ups. Int Endod J. 2003; 36 (7): 453 - 463.

17. Yoldas O, Topuz A, Isci AS, Oztunc H. Postoperative pain after endodontic retreatment: single- versus two-visit treatment. Oral Surg Oral Med Oral Pathol Oral Radiol Endod. 2004; 98 (4): 483 - 487. 
18. Alacam T, Tinaz AC. Interappointment emergencies in teeth with necrotic pulps. J Endod. 2002; 28 (5): 375 - 377.

19. Wang C, Xu P, Ren L, Dong G, Ye L. Comparison of postobturation pain experience following one-visit and twovisit root canal treatment on teeth with vital pulps: a randomized controlled trial. Int Endod J. 2010; 43 (8): 692 - 697.

20. Ghivari SB, Kubasad GC, Chandak MG, Akarte N. Apical extrusion of debris and irrigant using hand and rotary systems: A comparative study. J Conserv Dent. 2011; 14 (2): 187 - 190.

21. Madhusudhana K, Mathew VB, Reddy NM. Apical extrusion of debris and irrigants using hand and three rotary instrumentation systems- An in vitro study. Contemp Clin Dent. 2010; 1 (4): 234 - 236.

22. Basrani B. Irrigation in endodontic treatment. Alpha Omegan. 2011; 104 (1-2): 18 - 25.

23. Ehrmann EH, Messer HH, Clark RM. Flare-ups in endodontics and their relationship to various medicaments. Aust Endod J. 2007; 33 (3): 119 - 130.

24. Contardo L, Meneguzzi E, Cadenaro M, Di Lenarda R. Clinical evaluation of antibiotic prophylaxis before endodontic treatment of necrotic teeth. Minerva Stomatol. 2005; 54 (3): 153 - 160.

25. Balto K. Single- or multiple-visit endodontics: which technique results in fewest postoperative problems? Evid Based Dent. 2009;10 (1): 16.

26. Wu MK, Wesselink PR, Walton RE. Apical terminus location of root canal treatment procedures. Oral Surg Oral Med Oral Pathol Oral Radiol Endod. 2000; 89 (1): 99 - 103.

Correos electrónicos de los autores:

Claudia Liliana Martínez Vera: odontomartinezvera@yahoo.es Liliana Rodríguez Ballesteros:

Antonio José Díaz Caballero: adiazc1@unicartagena.edu.co

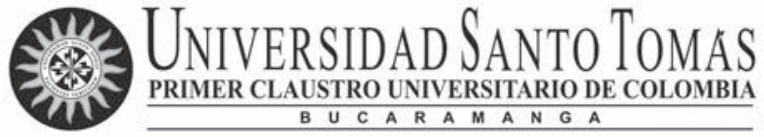

\section{Facultad de Odontología}

SNIES 1097 - Acreditación de Alta Calidad 517 del 6 de febrero de 2008 - Duración: 10 semestres
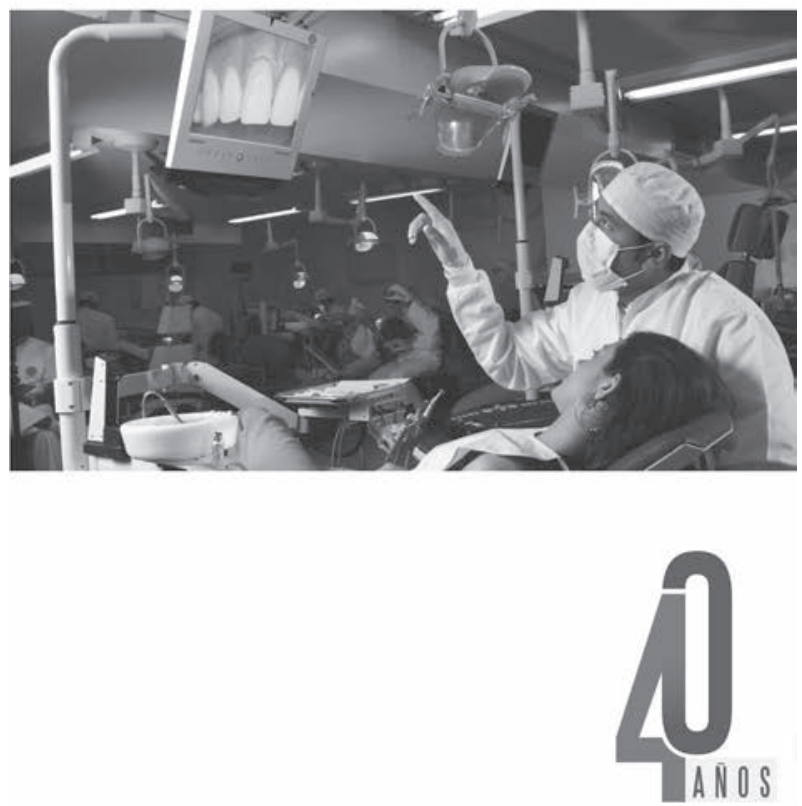

INSCRIPCIONES ABIERTAS

Teléfono: 6800801

ext: 1263 - $1264-1265-1801$

Línea Gratuita 018000917044

promocion@mail.ustabuca.edu.co

BUCARAMANGA

\section{www.ustabuca.edu.co}

